\title{
Status of the Measurement of Neutrino-Electron Elastic Scattering in the NOvA Near Detector
}

Don Athula Wickremasinghe (Fermilab) for the NOvA Collaboration

\begin{tabular}{|c|c|}
\hline Introd & \\
\hline$\nu_{e}+e^{-} \rightarrow \nu_{e}+e^{-}$ & $\nu_{\mu}+e^{-} \rightarrow \nu_{\mu}+e$ \\
\hline
\end{tabular}

Neutrino-electron elastic scattering is a leptonic process which exchanges a vector boson to scatter off an electron.

According to the standard model (SM), the cross section has been calculated as a function of the neutrino energy:

$$
\sigma^{\nu_{l} e \rightarrow \nu_{l} e} \sim 10^{-42}\left(E_{\nu} / \mathrm{GeV}\right) \mathrm{cm}^{2} \quad \text { ( Uncertainty } \sim 1 \% \text { ) }
$$

Measuring neutrino-electron scattering is an experimentally challenging task due to the smallness of the scattering cross section.

* Since, there is no hadronic or nuclear uncertainties associate with this process, the neutrino-electron scattering measurement is an important tool to constrain the neutrino flux prediction to reduce the total uncertainty.

\section{NOvA Near Detector}

* NOvA ( NuMI Off-Axis $\nu_{e}$ Appearance Experiment ) is a long baseline $(800 \mathrm{~km})$ neutrino experiment to observes the oscillation of muon neutrinos to electron-neutrinos

* The near detector (ND) has been placed $1 \mathrm{~km}$ away from the NuMI target to match $14.6 \mathrm{mrad}$ off axis spectrum at the far detector from the beam

* ND is located $100 \mathrm{~m}$ underground at Fermilab

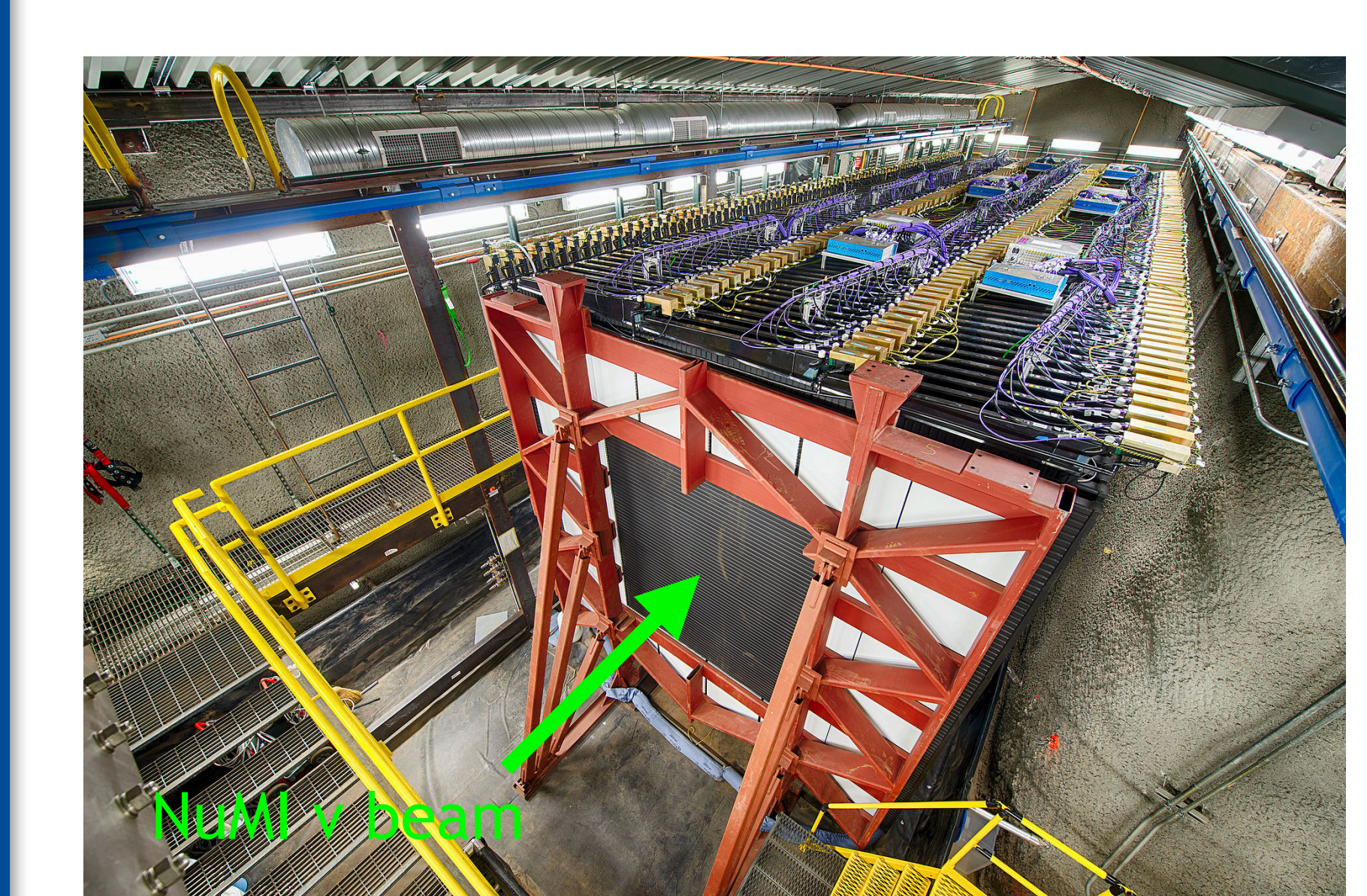

$*$ ND is a tracking calorimeter with $\mathbf{3 0 0}$ ton liquid scintillator * ND has 18,000 channels * The high rate of neutrino interactions at the ND provides opportunities to make detailed measurements of neutrinonucleus cross sections

\section{Event Identification}

According to the kinematics of $\nu-e$ scattering, the relationship of the scattering angle of electron $\left(\theta_{e}\right)$ with the neutrino energy $\left(E_{\nu}\right)$ and electron energy $\left(E_{e}\right)$ is: $1-\cos \theta_{e}=\frac{m_{e}\left(1-T_{e} / E_{\nu}\right)}{E_{e}}$ where: $T_{e}$ : Electron Kinetic Energy .

This sets the limits for the signature of the $\nu-e$ as: $E_{e} \cdot \theta_{e}^{2}$ to be small $\left(<2 m_{e}\right)$

Therefore, we are searching for events as:

* Very forward going single prong events with small $E_{e} \cdot \theta_{e}^{2}$ peaking around zero

* The Convolutional Visual Network (CVN) score for electron identification should be above 0.8

* Reconstructed hadron energy should be small

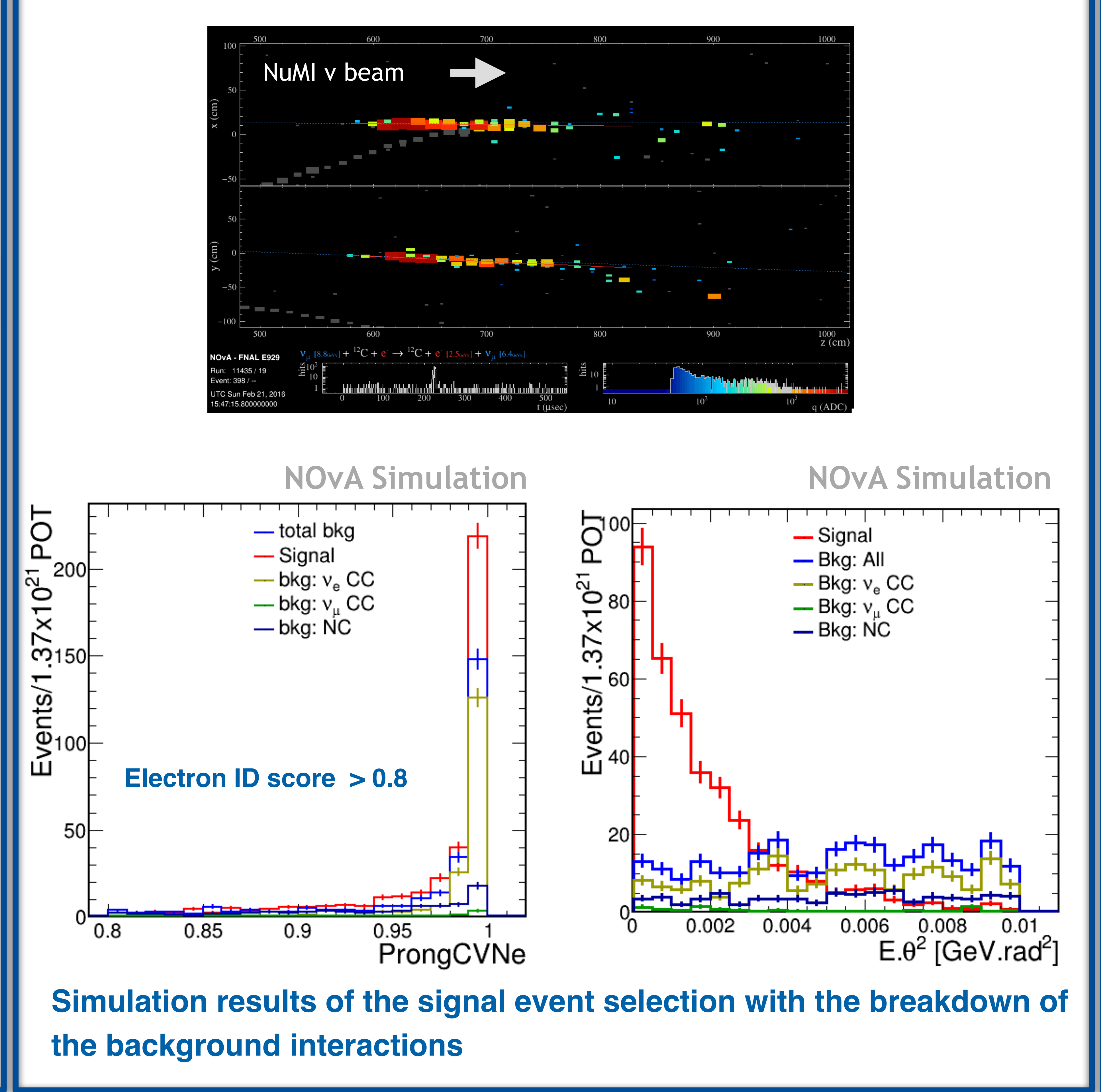

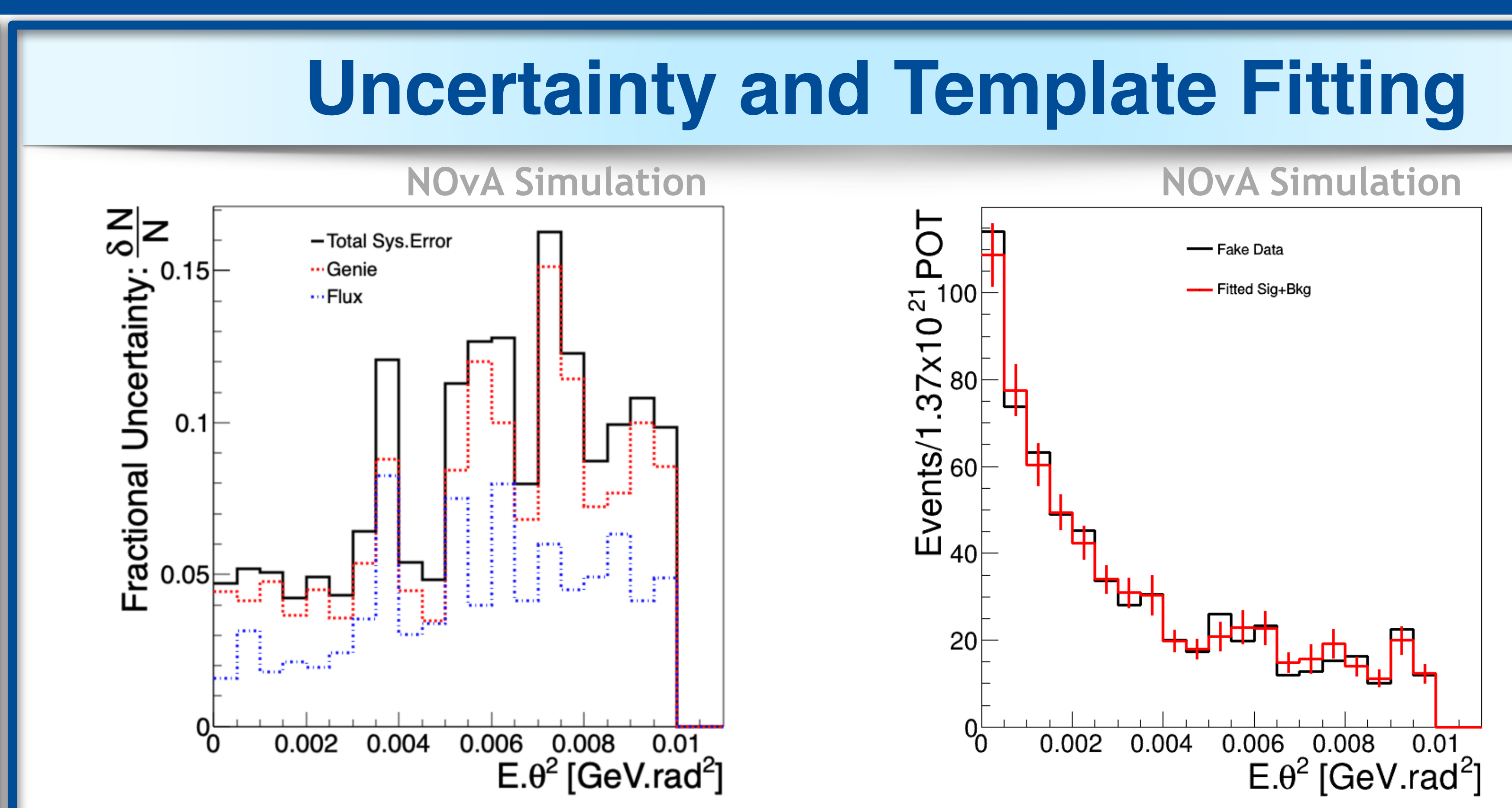

* $\nu-\mathrm{A}$ and FSI modeling systematic uncertainty and neutrino flux uncertainties have been taken into account to the total uncertainty calculation

* The detector response uncertainties will be taken into account for the final uncertainty estimations in the future

* A template fitting algorithm has been tested to fit fake data on the total Signal + Background distribution of $E_{e} \cdot \theta_{e}^{2}$

* The fake data for this study has been randomly chosen within the total uncertainty

* The template fitting is performed by using a defined $\chi^{2}$ with taking account the total covariance matrix (COV) to obtain the normalization factors for the signal (Sig) and the background (Bkg) $\chi^{2}=\sum[\text { Data }-([0] . \text { Sig }+[1] . \text { Bkg }))_{\mathbf{i}} \cdot \operatorname{COV}_{\mathbf{i j}}^{-1}(\text { Data }-([0] . S i g+[1] . \mathbf{B k g})]_{\mathbf{j}}$

\section{Conclusion}

* A method [1] of applying Bayes theorem by taking account the observations and the model predictions is a possible method to model predictions
constrain the flux

* Previous studies indicate that the NOvA flux normalization uncertainty can be reduced by at least $40 \%$ using neutrinoelectron scattering events in the NOvA near detector

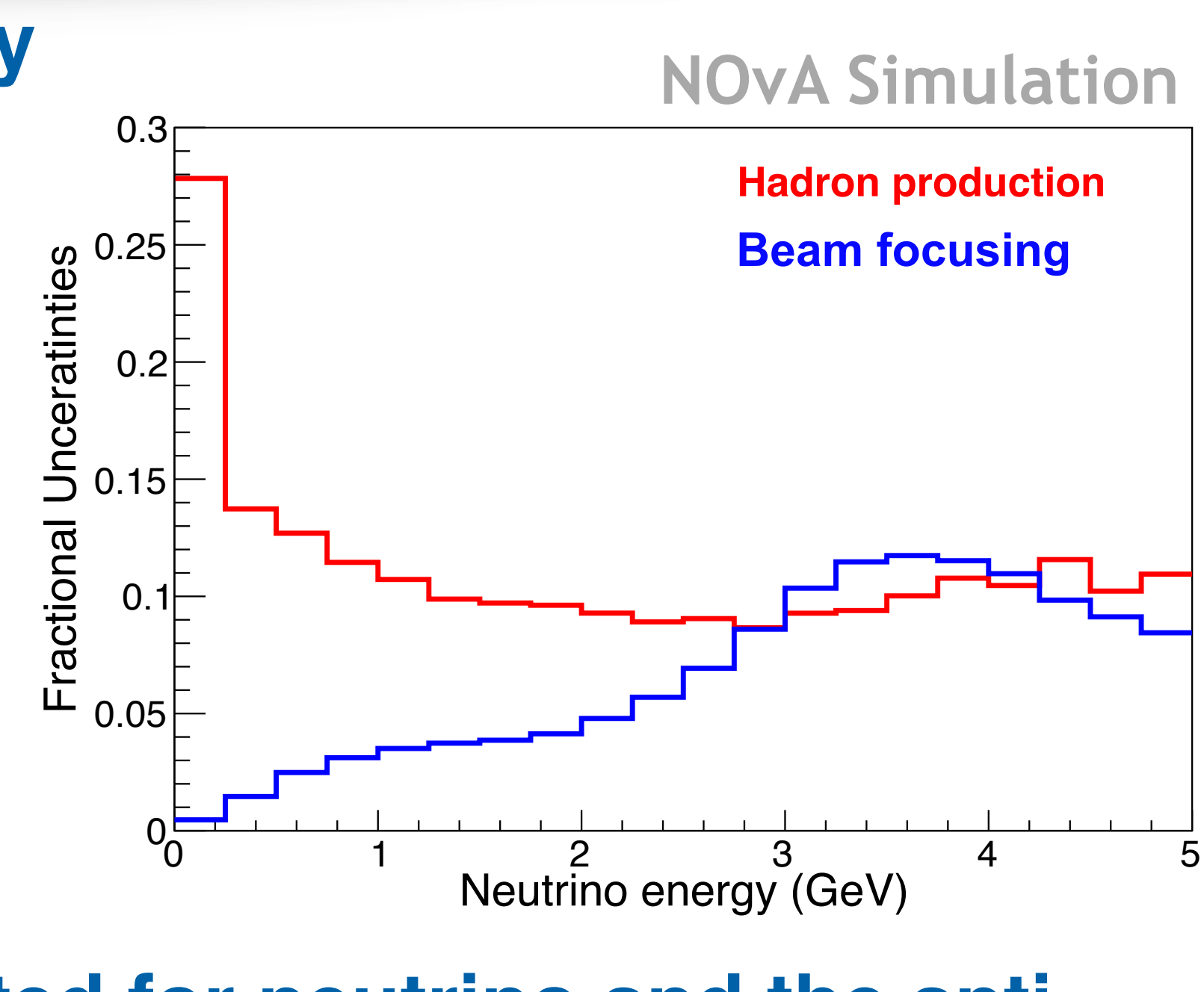

The combined results from the data collected for neutrino and the antineutrino modes will be a powerful flux constraint tool

* Also this measurement could be used to constrain new physics such as the magnetic moment of the neutrino

[1] MINERvA Collaboration, "Measurement of neutrino flux from neutrino-electron elastic scattering”, Phys.Rev.D93 (2016) 11, 112007 